\title{
Origin and speciation of Picea schrenkiana and Picea smithiana in the Center Asian Highlands and Himalayas
}

\author{
Lili Li • Yongshuai Sun • Jiabin Zou • Wei Yue • \\ Xi Wang • Jianquan Liu
}

Published online: 17 August 2014

(C) The Author(s) 2014. This article is published with open access at Springerlink.com

\begin{abstract}
Elucidating the evolutionary history of current species diversity, especially trees with large effective population sizes and long generation times, is a complicated exercise confounded by gene flow and incomplete lineage sorting. In the present study, we aim to determine the origin and speciation of Picea schrenkiana and Picea smithiana using population genetic data from chloroplast (cp), mitochondrial (mt), and nuclear (nr) genomes. These two species occur in the Central Asian Highlands and Himalayas, respectively, where they are isolated from other Asian congeneric species by the Qinghai-Tibet Plateau (QTP) or adjacent deserts. Previous studies based on both morphological and molecular evidence suggest that they have contrasting phylogenetic relationships with Picea likiangensis or Picea wilsonii which are closely related and both located in the QTP. We examined genetic variation among 16 loci of three genomes from 30 populations of these four species. At both cpDNA loci and mtDNA loci, $P$. schrenkiana appeared to be closely related to P. likiangensis, although statistical support for this was weak. However, phylogenetic analyses and speciation tests based on the nuclear data from 11 loci provided evidence that P. schrenkiana and P. smithiana are sister species. These two species diverged around five million years ago (Mya) while the divergence between them and the P. likiangensisP. wilsonii clade occurred about 18.4 Mya. We also detected gene flow accompanying these speciation events. Our results highlight the complex speciation histories of these alpine conifers due to interspecific gene flow and/or incomplete
\end{abstract}

Electronic supplementary material The online version of this article (doi:10.1007/s11105-014-0774-5) contains supplementary material, which is available to authorized users.

$\mathrm{L}$. Li $\cdot$ Y. Sun $\cdot$ J. Zou $\cdot$ W. Yue $\cdot$ X. Wang $\cdot$ J. Liu $(\bowtie)$

State Key Laboratory of Grassland Agro-Ecosystems, School of Life

Sciences, Lanzhou University, Lanzhou 730000, China

e-mail: liujq@1zu.edu.cn lineage sorting, and the importance of the early QTP uplifts in promoting the origin of these important conifer species in the Asian highlands.

Keywords Gene flow $\cdot$ Picea schrenkiana $\cdot$ P. smithiana . Population genetic data $\cdot$ Speciation test

\section{Introduction}

Geographical or other selective barriers to genetic exchange may give rise to new species by isolating previously interbreeding populations (Coyne and Orr 2004). Such geographical isolation can play an important role in dividing a common ancestor into two (rarely more) sister species in which different random alleles become fixed and adaptions to different local conditions are selected (Mayr 1954; Grant 1981; Coyne 1992; Orr and Presgraves 2000). In addition, gene flow and incomplete lineage sorting complicate speciation process, especially in tree species with large effective population sizes and long generation times (Gavrilets 2003; Levin 2003; Rieseberg and Willis 2007). Evidence increasingly suggests that population-genetic data, derived from sequencing multiple loci, is the best approach for clarifying the origin and speciation of such tree species (e.g., Chen et al. 2010; Zou et al. 2013; Sun et al. 2014). Such data allow for alternative speciation models and estimation of the extent of gene flow that has accompanied speciation (e.g., Hey and Nielsen 2004, 2007; Hey 2006, 2010a, b; Li et al. 2010). In addition, estimates of divergence timescales based on analysis of multilocus population genetic data in a coalescent framework provide good temporal hierarchies for understanding the roles of geological events in triggering speciation (Wakeley 2003; Takayama et al. 2013).

Numerous alpine plant species are endemic in the QinghaiTibet Plateau (QTP), the Himalayas, and the Asian 
highlands - regions which together are recognized as one of the world's most important alpine biodiversity hotspots (Wilson 1992; Myers et al. 2000). These endemic species may have originated during and/or after the extensive uplifts of the QTP and Himalayas. However, the date when both the QTP and Himalayas were uplifted to their current heights remains debatable, three extensive uplifts have been dated between 22 and 20, 15-8 and 5-3 Mya (e.g., Shi et al. 1998; An et al. 2001; Guo et al. 2002; Buslov et al. 2007; Wang et al. 2008). Previous studies have found that a few herbaceous genera diversified greatly during these three stages, especially the latter two (e.g., Liu et al. 2002, 2006; Wang et al. 2009). Studies designed to examine the origin and speciation of alpine trees, especially studies based on population genetic data, are rare (but see Mao et al. 2010 and Xu et al. 2010). In the present paper, we examine the phylogenetic relationships and speciation patterns of two Asian highland spruce species: Picea schrenkiana and Picea smithiana. The genus Picea diversified greatly in Asia with 24 out of the total of 35 spruce species occurring there (Fu et al. 1999; Farjón 2001). Most of them are distributed in cold and temperate mountainous regions, especially in the QTP and adjacent highlands. However, P. schrenkiana and P. smithiana occur with narrow distributions in the Central Asian Highlands (Tian Shan Mountains; Zhang and Tang 1989) and the Himalayas, respectively. These two species are isolated from each other by the western Kunlun Mountains, and both are isolated from spruces in the QTP by the Himalaya and Tarim depressions. All these geographic isolations seem to have developed before the Pliocene (5.3-2.5 Mya; Fig. 1). For example, the QTPHimalayas uplift and Tarim depression occurred at the same as the first extensive uplift of the QTP around $22 \mathrm{Mya}$ (Guo et al. 2002). The second extensive QTP uplift, which occurred between 15 and 8 Mya, was accompanied by further increases of the western Kunlun Mountains and the Himalayas, as well as the Tarim depressions (Wang et al. 1990, 2006, 2008; Abdrakhmatov et al. 1996; Shi et al. 1998; An et al. 2001; Bullen et al. 2001, 2003; Charreau et al. 2006, 2009; Buslov et al. 2007; Dupont-Nivet et al. 2008). It is likely that the origin and speciation of $P$. schrenkiana and $P$. smithiana are correlated with geographical isolations consequent upon these geological events.

These two species are morphologically distinct from each other, despite having similar quadrangular leaves: $P$. schrenkiana usually has thick leaves while leaves of $P$. smithiana are linear and more slender. These two species were placed together in the section Picea (Liu 1982; Farjón 2001) along with other species (e.g., Picea wilsonii, Picea neoveitchii, Picea crassifolia, and Picea abies) with quadrangular leaves and stomatal lines present and almost equal in number on each surface (Fu et al. 1999). Those species represented by Picea likiangensis of Sect. Casicta (Liu 1982; Farjón 2001) have flattened leaves with stomatal lines mostly or only present on the adaxial surface. However, phylogenetic analyses based on chloroplast (cp) DNA sequence variations suggest that both $P$. schrenkiana and $P$. smithiana cluster together with $P$. likiangensis into a well-supported subclade, sister to that represented by $P$. wilsonii. The remaining species of Sect. Picea form a separate clade with distant relationships (Ran et al. 2006; Bouillé et al. 2011). In contrast, phylogenetic analyses based mainly on variations in the mitochondrial (mt) genome suggest that $P$. schrenkiana clusters into a subclade with $P$. wilsonii while $P$. smithiana should be placed at the basal position of the subclade containing $P$. likiangensis (Lockwood et al. 2013). Another recent phylogenetic analysis based on 11 concatenated nuclear gene sequences suggested that $P$. schrenkiana and P. smithiana clustered into a separate clade, sister to the other one comprising the other eight species (Sun et al. 2014). It should be noted that all other species, i.e., Picea spinulosa, Picea farreri, Picea neoveitchii, Picea morrisonicola, Picea purpurea, and Picea brachytyla are closely related to $P$. likiangensis or $P$. wilsonii, or are derived from an original hybrid of these two key species (Liu 1982; Fu et al. 1999; Ran et al. 2006; Bouillé et al. 2011; Lockwood et al. 2013; Zou et al. 2013; Sun et al. 2014). We therefore chose $P$. likiangensis and $P$. wilsonii to represent the other species and further examined their phylogenetic relationships with $P$. schrenkiana and P. smithiana based on three genomes data. We sequenced three cpDNA loci, two mtDNA loci and 11 nuclear DNA loci from 30 populations comprising 330 individuals of four species. We used approximate Bayesian computation (ABC) methods (Wegmann et al. 2010) to test alternative speciation hypotheses between these four species, estimate the divergence timescales, and examine whether gene flow occurred during speciation events.

\section{Material and Methods}

Population Sampling Populations of four species were sampled from across their major distributions and three varieties recognized under P. likiangensis were included (Fig. 1; Supplementary Table 1). The number of sampled individuals in each population was between 4 and 21 , spaced $\geq 100 \mathrm{~m}$ apart. A total of 330 individuals were used in this study. Fresh leaf needles were dried and stored in the field using silica gel; seeds were stored at $-20{ }^{\circ} \mathrm{C}$. The latitude, longitude, and altitude of each sampling location were measured using an Extrex GIS monitor (Germany) (Supplementary Table 1). One Picea meyeri individual was used as an outgroup for phylogenetic analyses.

Sequencing and Phasing We used either the modified cetyltrimethylammonium bromide (CTAB) procedure (Doyle and Doyle 1990) or a QIAGEN DNeasy Plant Mini Kit (QIAGEN, Valencia, CA, USA) to extract the total 
Fig. 1 Distributions of P. schrenkiana, P. smithiana, P. likiangensis, and $P$. wilsonii. $P$. schrenkiana distributed in Tian

Shan Mountains and neighboring areas (TSMs), P. smithiana distributed in Himalaya Mountains, $P$. likiangensis and $P$. wilsonii distributed in QinghaiTibet plateau and adjacent regions (QTPs). Different colors represent different species. The mean altitudes of mountains are given in Arabic numerals. Extensive geographical barriers, including deserts, are indicated by the areas of black dots; high mountains between the TSMs and the QTPs are shown by small triangles

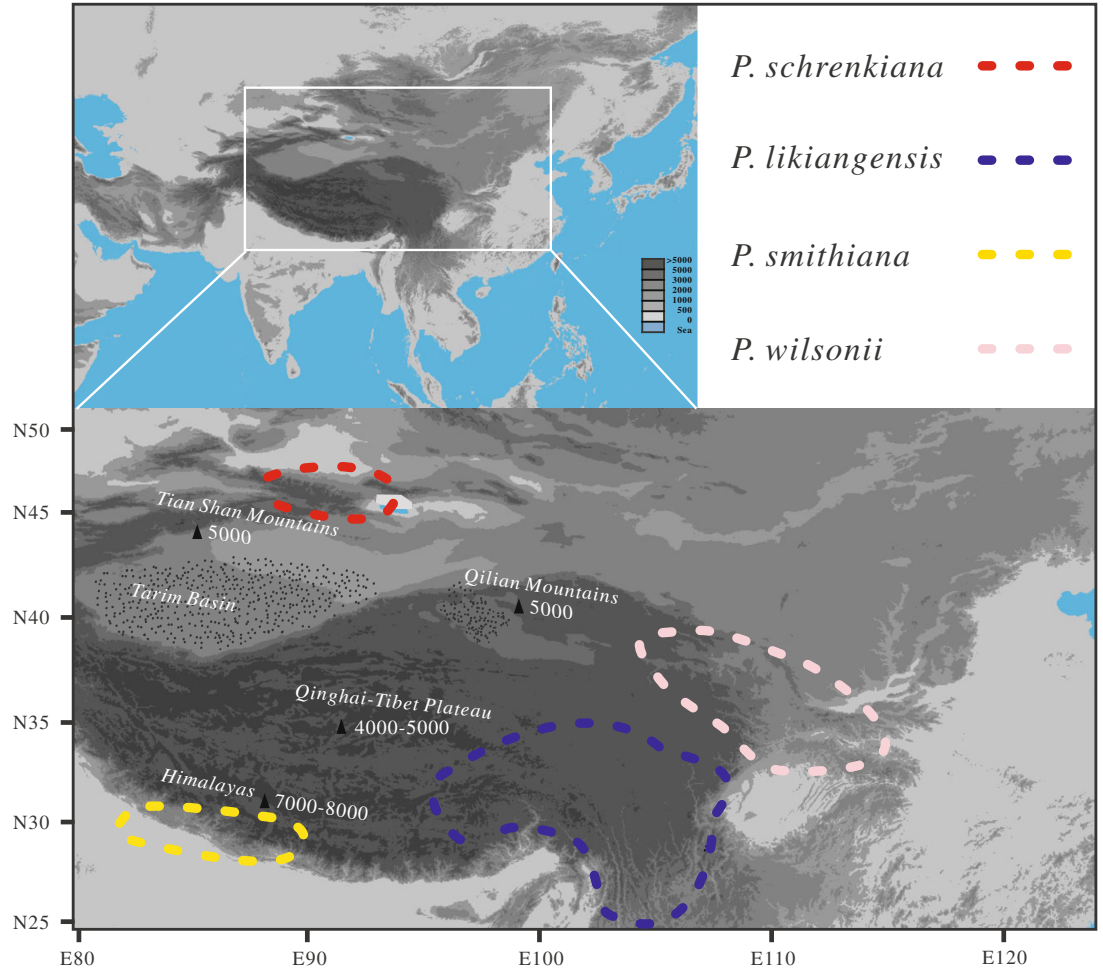

genomic DNA from needles. Eleven nuclear loci (4CL, EBS, GI, MOO2, M007D1, Sb16, Sb29, Sb62, se1364, se1390, and $x y 1420$ ) were selected and sequenced following $\mathrm{Li}$ et al. (2010) using an ABI 3130xl or 3730xl Genetic Analyzer (Applied Biosystems, Foster City, CA, USA) and an ABI Prism BigDye Terminator Cycle V3.1 Sequencing Kit. We amplified and sequenced three chloroplast loci $\operatorname{trn} \mathrm{L}-\operatorname{trn} \mathrm{F}$, $\operatorname{trn} \mathrm{S}-t r n \mathrm{G}$, and $n d h \mathrm{~K} / \mathrm{C}$; Taberlet et al. 1991; Hamilton 1999; Anderson et al. 2006) and two mitochondrial loci (nad1 intron b/c and nad5 intron1; Meng et al. 2007). Basecalling of nuclear genotypic sequences was done using Phred v0.020425.c (score >20) (Ewing et al. 1998) with CODONCODE ALIGNER software (CodonCode Corporation) and manually checked whether the SNPs consistent with the chromatogram peaks using the MEGA5 (Tamura et al. 2011). Sequences from $\mathrm{cp}$ and $\mathrm{mt}$ genomes were identified and checked using MEGA5. Finally, we used CLUSTAL W (Thompson et al. 1997) in MEGA5 to perform the alignment of sequence matrix at each locus.

Nuclear sequences with heterozygous sites were rephased and separated into two allele sequences by PHASE (Stephens et al. 2001, 2003) in the software package DnaSP V5 (Librado and Rozas 2009). A Hardy-Weinberg equilibrium was assumed and the phased sequences were inferred using a coalescent Bayesian method. Homozygous genotypes, and genotypes with single heterozygous sites, were set as known alleles to improve the performance of PHASE analyses. A general recombination model (Li and Stephens 2003) and 10 iterations of the final run were used and other optional parameters were set as default. Runs were repeated twice with different seed numbers to ensure that results were robust. In this research, three nuclear fragments (Sb16, Sb29, and Sb62,) were cloned and 10 clones were sequenced.

All newly obtained sequences of $P$. schrenkiana and P. smithiana were submitted to GenBank (accession numbers KJ176997-KJ179244) and three genomes sequences of P. likiangensis and P. wilsonii were cited from $\mathrm{Li}$ et al. (2013) and Zou et al. (2013).

Phylogenetic Analyses For cpDNA, mtDNA, and nuclear DNA, we used NETWORK V4.2.1.1 (Bandelt et al. 1999) (available at http://www.fluxus-engineering.com/) to construct their genealogies. We further used a Bayesian coalescent-based method for species tree estimation based on three genomes data, as implemented in BEAST 1.7.2 package (Drummond and Rambaut 2007; available at http:// beast.bio.ed.ac.uk/Main Page). The approach makes use of multilocus nuclear data, embedding individual gene trees in a multilocus species tree, allowing estimation of species genealogy (Hailer et al. 2012). Nonrecombining blocks of each locus were chosen using IMGC software (Woerner et al. 2007) because recombination cannot be modeled in the coalescence approach in BEAST. Using a strict molecular clock and the models of sequence evolution indicated by jModeltest (Posada 2008), we performed three independent runs of $2 \times 10^{8}$ generations and sampled parameters every 5,000 iterations, with a burn-in of $30 \%$. Convergence was checked in Tracer 1.4 (available at http://beast.bio.ed.ac.uk/ 
Tracer). For both cpDNA and mtDNA, all indels were coded as " 0 " and "1". Branch supports were also evaluated using the program MrBayes 3.1.2 (Huelsenbeck et al. 2001; Ronquist and Huelsenbeck 2003).

Population Genetic Parameters and Neutrality Tests We used DnaSP V5 (Librado and Rozas 2009) to calculate basic population genetic parameters: the number of segregating sites $(S)$, Watterson's parameter $\left(\theta_{w}\right.$, Watterson 1975), nucleotide diversity ( $\pi$, Tajima 1983). For nuclear loci, we further showed the divergence between each pair of species using ARLEQUIN, version 3.0 (Excoffier et al. 2005) with significance tests based on 10,000 permutations.

Before estimating evolutionary history, we used a recently developed maximum frequency of derived mutation (MFDM) method ( $\mathrm{Li} 2011$ ) to test the neutrality of variation at each locus. The MFDM method is not affected by the impact of varying population size, such as expansion or shrinkage, which may produce similar signals to selection (Li 2011). Because the demographic dynamics cannot change the genealogies of a locus (Tajima 1983; Hein et al. 2004), an unbalanced topology shaped by recent selection could be captured and stand as evidence of nonneutrality. Simulation analysis and comparisons with several other methods showed higher power and a lower falsepositive rate when detecting recent selection using the MFDM method (Li 2011). Here, we tested the neutrality per locus in each species with this method using a $5 \%$ significance level. Ancestral states at variable sites were determined by comparison with two outgroup species (Picea breweriana and P. meyeri).

Linkage Disequilibrium and Population Structure Linkage disequilibrium (LD) was measured by $r^{2}$, the square of the correlation coefficient between each single nucleotide polymorphism (SNP) pair (Heuertz et al. 2006), using DnaSP V5 (Librado and Rozas 2009). The significance level of the statistical association between alleles at different sites was measured using Fisher's exact test, and Bonferroni correction was used to correct for false positives. $r^{2}=0$ means loci are in complete linkage equilibrium and $r^{2}=1$ indicates loci are in complete linkage disequilibrium. However, the result showed that $r^{2}$ values were very small, almost equal to zero, which indicated loci were nearly no linkage disequilibrium (Supplementary Table 2). Therefore, we used all SNPs of nuclear loci to identify the genetic structure and inter relationships of four species at the population level by STRUCTURE V2.3.4 analysis (Pritchard et al. 2000; Hubisz et al. 2009). The most likely number of populations in the dataset $(K)$ was estimated by conducting 15 independent runs for each $K$ value ranging from 1 to 6 . Each run had a burn-in of 200,000 iterations and additional 500,000 iterations. Finally, we used Distruct v.1.1 (Rosenberg 2004) to draw the graphics. We computed $\Delta K$, using the method in Evanno et al. (2005), to determine the most likely number of clusters.

Tests for Speciational Bottlenecks and Gene Flow Using ABCtoolbox We compared four speciation models with and without gene flow using Approximate Bayesian Computation (ABC) implemented in the ABCtoolbox software package (Wegmann et al. 2010) based on the 11 nuclear loci sequence data. We then used Bayesian factors (BF) to choose the more suitable of two compared models, if $\mathrm{BF}>3$, model $\mathrm{A}$ was better than model B or C (Fig. 2). We adopted four steps to test speciation orders between four species in order to reduce the number of the hypothesized models (together 64 models) and shorten modeling time (together more than 1 year). Firstly, we examined the interspecific relationships among P. schrenkiana, P. likiangensis, and $P$. wilsonii based on all three possible models. Secondly, we tested whether $P$. smithiana is closely related to $P$. likiangensis or $P$. wilsonii using a similar approach to the first step. Both tests from the first and second steps suggested that $P$. likiangensis and $P$. wilsonii always clustered together relative to P. schrenkiana or P. smithiana (Supplementary Table 3). Thirdly, we treated $P$. likiangensis $-P$. wilsonii as one clade, and examined its relationships with $P$. schrenkiana and $P$. smithiana. This test also suggested that $P$. smithiana and $P$. schrenkiana clustered together, diverging late from the $P$. likiangensis $-P$. wilsonii clade. Finally, we compared two different models with or without gene flow during these series of speciation events.

The current population sizes of $P$. schrenkiana, P. smithiana, $P$. likiangensis, $P$. wilsonii, and ancestor species were recorded as $N S C H, N S M I, N L, N W, N 1$ (ancestor of $P$. likiangensis and $P$. wilsonii), N2 (ancestor of P. schrenkiana and P. smithiana), and N3 (ancestor of all four species), respectively. The divergence time was recorded as $T 1, T 2$, and $T 3$, representing the divergence time between P. schrenkiana and P. smithiana, P. likiangensis and $P$. wilsonii, and $P$. likiangensis-P. wilsonii and $P$. schrenkiana-P. smithiana, respectively. $\mathrm{Nm}$ was used to represent the migration numbers per generation.

We assumed uniform priors on the $\log _{10}$ scale for all population sizes (for $N 1(4.5,5.7)$, for $N 2(3.8,5.0)$, for $N 3$ $(3.8,6.0)$, for $N L(5.3,5.5), N W(5.4,5.6)$, and for $N S C H /$ $N S M I(4.5,5.0))$ and three timing parameters (for $T 1$ (5.1, 5.3), for $T 2(5.2,5.5)$, and for $T 3(5.5,5.8))$. We used 34 statistics computed by ARLEQUIN v3 (Excoffier et al. 2005) to summarize the population genetic information of P. schrenkiana or P. smithiana together with P. likiangensis and $P$. wilsonii, and we used 34 statistics to estimate the speciation models of four species. Thus, for each species, we computed the number of segregating sites, the number of private segregating sites, Tajima's $D$ (Tajima 1989), Fu and Li's $D^{*}(\mathrm{Fu}$ et al. 1999), and the number of pairwise 
Fig. 2 Models for origin of P. schrenkiana $(\mathrm{SCH})$, P. smithiana (SMI),

P. likiangensis (LIK) and P. wilsonii (WIL). If $\mathrm{BF}>3$, model A was better than model $\mathrm{B} / \mathrm{C}$ or model $\mathrm{B}$ was better than model A. T1, T2, and T3 indicates the divergence time between $P$. schrenkian and P. smithiana, $P$. likiangensis and $P$. wilsonii, and $P$. likiangensis $-P$. wilsonii and $P$. schrenkiana-P. smithiana. The black arrows indicate the migrations between pairs of species

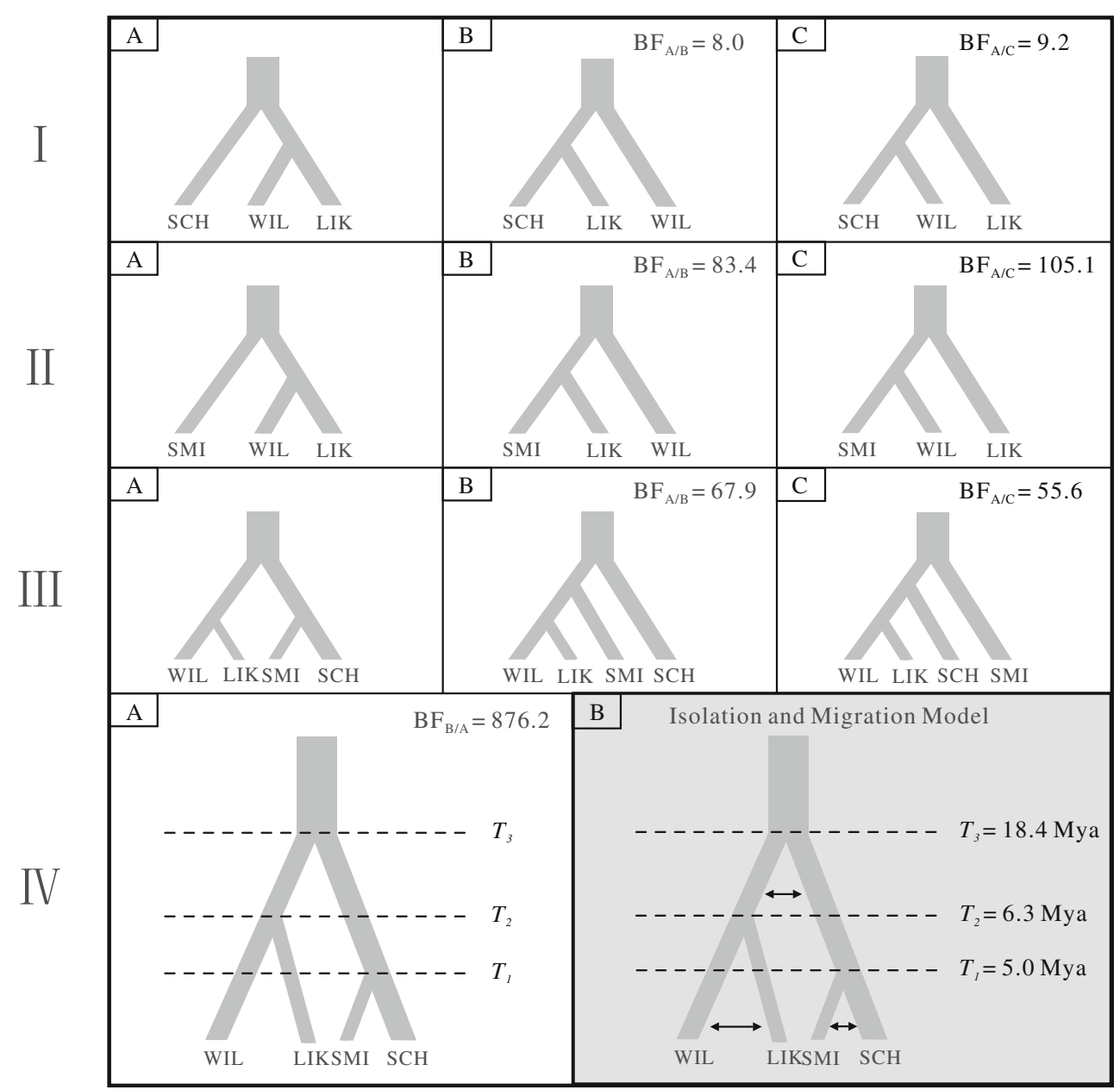

differences. For each pair of species, we computed $F_{\mathrm{ST}}$, the average number of pairwise differences and the significance was tested by 1,000 permutations as implemented in ARLEQUIN version 3.0 (Excoffier et al. 2005). We also computed the total number of segregating sites over both species. To decrease the redundancy of statistics, we extracted partial least squares (PLS) components from the total of 34 summary statistics using the specific R script of ABCtoolbox (Wegmann et al. 2009). A total of 28 PLS components were used according to root mean square error plots and the conversion equations were inferred from the 10,000 samples simulated by a standard algorithm for each of the last speciation models. We used the likelihood-free ABC-MCMC (Markov chain Monte Carlo) methods (Wegmann et al. 2009) and the program fastsimcoal (Excoffier and Foll 2011) to simulate a total of 1,000,000 samples with a proposed range of $\varphi=1$ and tolerance $\delta=1 \%$. The 10,000 simulated samples used for inferring PLS components were used as the calibration in the MCMC sampling step in this study. We retained the 10,000 simulated samples that compared closest with the observed summary statistics of actual dataset following the PLS transformations and applied the regression adjustment general linear model (GLM) to generate posterior distributions of all parameters in each model. Parameters were estimated based on a mean mutation rate of $\mu=1.41 \times 10^{-8}$ per site per generation, with a conservative confidence interval between an extremely "slow" rate $\left(1.11 \times 10^{-8}\right.$ per site per generation) and an extremely "fast" rate $\left(1.71 \times 10^{-8}\right.$ per site per generation). Average generation time was set to 50 years according to previous studies for spruce species (Bousquet and Bouillé 2005; Chen et al. 2010) and the dated timescales need further confirmation from independent evidence. This generation time is about three to five times to the age at the first reproduction, but less than the maximum life expectancy of spruce species (Burns and Honkala 1990). It is likely that alpine spruces in our studied region may have longer generation times than those distributed in the low-altitude region. Even if these demographic parameters are underestimated, our population genetic estimates are of the same order of magnitude and largely consistent with those from other studies of spruce species (Bousquet and Bouillé 2005; Chen et al. 2010; Li et al. 2010). To detect gene flow after speciation, we further used IMa2 (Hey 2010a, 2010b) to estimate the interspecific gene flow after divergence based on the isolation-with-migration 
(IM) model (Nielsen and Wakeley 2001; Hey and Nielsen 2004, 2007; Hey 2010a, 2010b).

Demographic Expansion Analysis To assess the demographic history of four spruce species, we used LAMARC v2.1.8 (Kuhner 2006; available at http://evolution.genetics. washington.edu/lamarc/index.html), with a coalescent simulation approach that considered the genealogical relationships among the nuclear haplotypes to estimate the exponential population growth rate parameter $(g)$ within the two species. The analysis was based on a Bayesian method, using Metropolis-coupled MCMC with replication of chains and adaptive heating to obtain better sampling of the parameter space. Large, positive values of the exponential growth parameter $(g)$ indicate population expansion, but negative values indicate population shrinkage while relatively small positive values $(g=10)$ may indicate little or no growth.

\section{Results}

Variation in Chloroplast and Mitochondrial Loci and Interspecific Relationships A total of 11 chlorotypes were identified based on the concatenated data of three chloroplast loci $(t r n \mathrm{~L}-t r n \mathrm{~F}, \operatorname{trn} \mathrm{S}-t r n \mathrm{G}$, and $n d h \mathrm{~K} / \mathrm{C})$ across all individuals of the four spruce species (Supplementary Table 4). Chlorotypes of four species were grouped into two distinct groups and no haplotype was shared between species (Fig. 3). Two haplotypes from $P$. wilsonii comprised a single distinct clade with six steps detected. The other comprised six hapltoypes (C6C11) from $P$. smithiana, a single haplotype $\mathrm{C} 5$ from $P$. schrenkiana, and two (C2 and $\mathrm{C} 1)$ from $P$. likiangensis. $\mathrm{C} 5$ appeared to be more closely related to $\mathrm{C} 2$ and $\mathrm{C} 1$ than to C6-C11 in the network. Phylogenetic analyses also identified two distinct clades, and close relationships between haplotypes from $P$. schrenkiana and $P$. likiangensis were not supported by the statistics (Fig. 4).

Fourteen mitotypes were identified across all samples from the examination of the sequence variation based on concatenated data of two mitochondrial loci (nad5 intron 1 and nadl intron b/c; Supplementary Table 5). No mitotype was shared between any two species. Similarly, two distinct groups were identified according to both network and phylogenetic analyses. Four mitotypes M6 to M9 from P. wilsonii (Fig. 3) comprised one clade at five steps from the other three species. In the other clade, two mitotypes (M10 and M11) in $P$. schrenkiana appeared to be closer to those (M1 to M5) found in P. likiangensis although such a relationship was not well supported (Fig. 4).

Variation in Nuclear Loci and Interspecific Relationships We sequenced 11 unlinked nuclear loci with a total concatenated length of 4,986 bp. We revealed 32, 38, 133, and 143 segregating sites in $P$. schrenkiana, $P$. smithiana, $P$. likiangensis, and $P$. wilsonii, respectively. $P$. schrenkiana and $P$. smithiana had a low level of silent nucleotide diversity, $\pi_{s}(0.0012)$ and $\pi_{s}$ (0.0016). A similar pattern was observed for the total nucleotide diversity (Table 1). P. likiangensis showed the highest nucleotide diversity in cytoplasmic DNA. MFDM

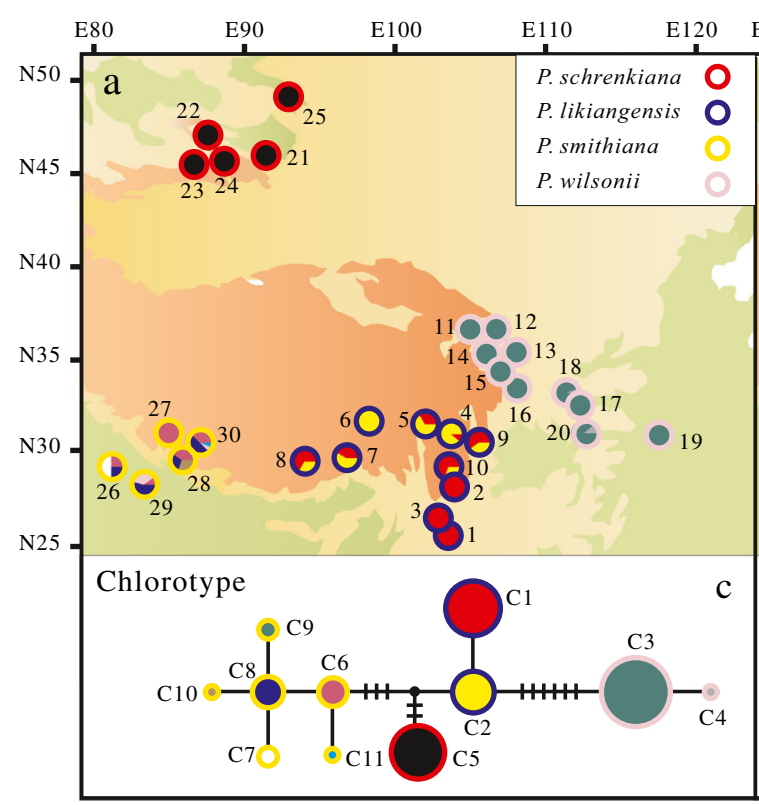

Fig. 3 Distributions (a, b) and networks (c, d) of chlorotypes and mitotypes within four species: $P$. schrenkiana, $P$. smithiana $P$. likiangensis, and $P$. wilsonii. The color of the circumference of the circles indicates each species. The proportion of each colored sector filling the

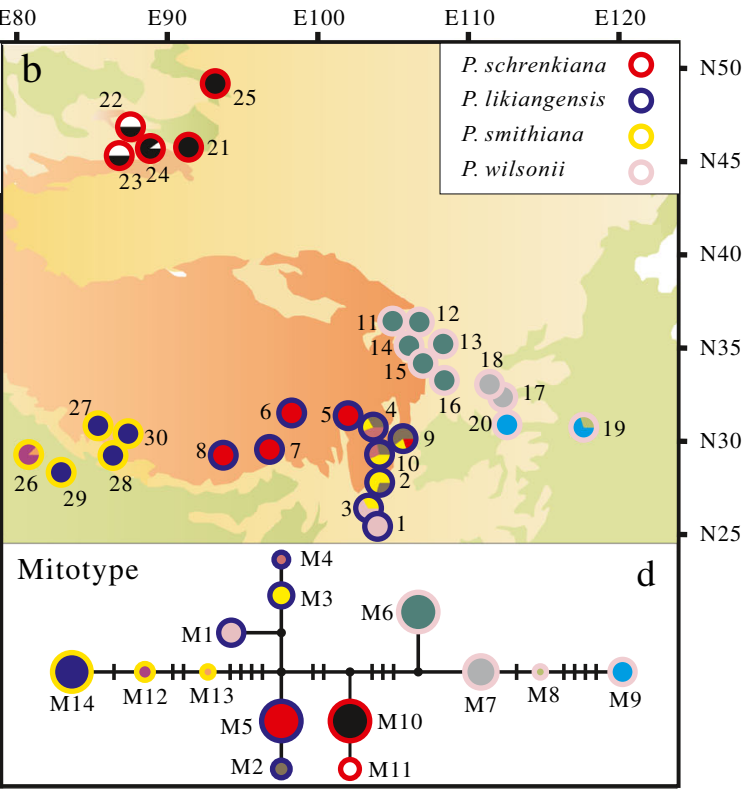

circles in $\mathbf{a}$ and $\mathbf{b}$ indicates the frequency of each chlorotype or mitotype in each species. Each haplotype in $\mathbf{c}$ and $\mathbf{d}$ is represented by a circle whose size is proportional to its frequency over all populations 
Fig. 4 Phylogenetic trees constructed for chlorotypes (a) and mitotypes (b) recovered from all sampled individuals among four spruce species. Numbers above nodes indicate statistical significance

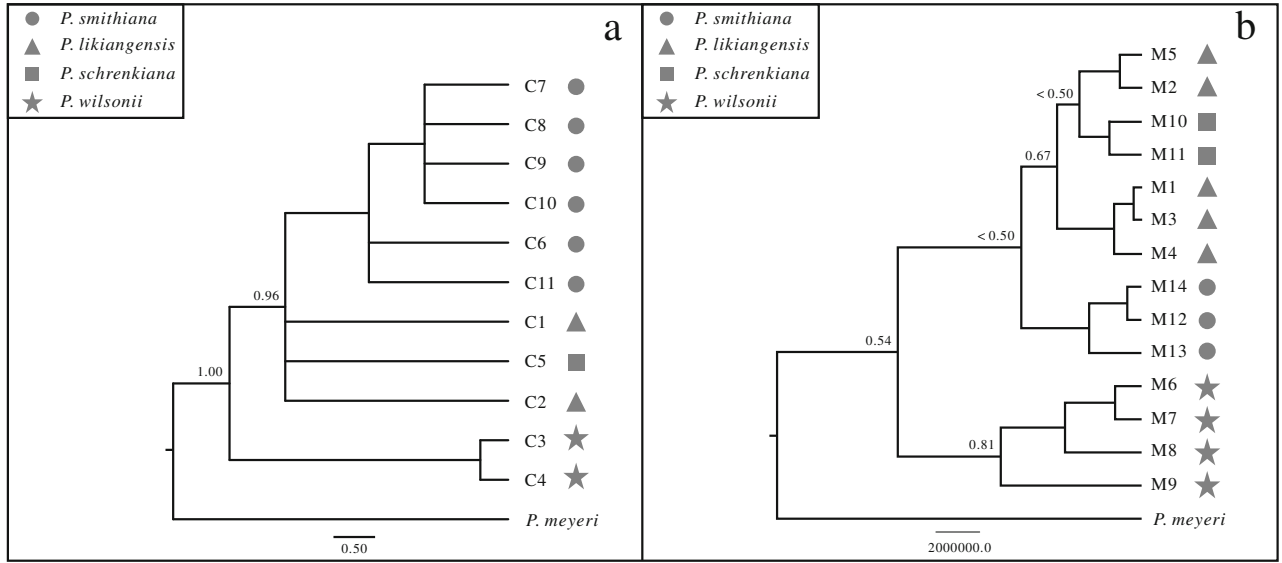

tests revealed nearly no substantial evidence for selection acting on each locus across all four species (Table 2).

Eight haplotype genealogies constructed by NETWORK V4.2.1.1 are shown in Supplementary Fig. 1, and the remaining three are not shown because of too many haplotypes. P. schrenkiana was highly differentiated from the other four species (se1390, se1364, Sb29, and MOO2), while $P$. smithiana could be distinguished from the other species at only three loci (se1390, GI, and 4CL). At the remaining loci, interspecific differentiations were low and indistinct. However, AMOVAs conducted on all nuclear data showed that divergence between each pair of species was highly significant $(P<0.001$, Table 3$)$ and the highest genetic divergence existed between $P$. schrenkiana and $P$. smithiana (Table 3) which may be caused by large geographic distance.

We then used a Bayesian clustering algorithm (Structure version 2.3.4) to construct the genetic structures of all examined populations and individuals. The $\Delta K$ (Evanno et al. 2005) tests showed that the most likely number of groups
$(K)$ for the entire dataset, using the total nuclear sequence data, was $K=4$ and that the individuals of all four species clustered into four distinct clusters with high probability. In the clustering results for $K=2$, the first cluster contained exclusively individuals from $P$. schrenkiana and P. smithiana, while the second cluster contained $P$. likiangensis and $P$. wilsonii. When $K=3, P$. schrenkiana and $P$. smithiana still clustered into one group, but $P$. likiangensis and $P$. wilsonii clustered into two separate groups (Fig. 5, Supplementary Fig. 2). The phylogenetic tree based on the concatenated sequences of all nuclear loci indicated $P$. schrenkiana and P. smithiana clustered to one clade, and $P$. likiangensis and $P$. wilsonii formed the other with high-supporting values (Fig. 6). However, the individuals from $P$. likiangensis and $P$. wilsonii did not cluster into separate subclades, probably due to gene flow or intraspecific substructure.

Tests of Speciation Models and Demographic Histories of Four Species The simulations with two demographic models

Table 1 Average nucleotide diversity across three different genomes in each of the four spruce species

\begin{tabular}{|c|c|c|c|c|c|c|c|c|c|c|c|c|c|c|}
\hline \multirow[t]{2}{*}{ Genomes } & \multirow[t]{2}{*}{ Species } & \multicolumn{5}{|c|}{ Total } & \multicolumn{4}{|c|}{ Nonsynonymous sites } & \multicolumn{4}{|c|}{ Silent sites } \\
\hline & & $n$ & $L$ & $S$ & $\theta_{W}$ & $\pi$ & $L$ & $S$ & $\theta_{W}$ & $\pi$ & $L$ & $S$ & $\theta_{W}$ & $\pi$ \\
\hline \multirow[t]{4}{*}{ nrDNAs } & P. schrenkiana & 60 & 4,986 & 32 & 0.0014 & 0.0012 & $2,444.37$ & 14 & 0.0012 & 0.0012 & $2,532.63$ & 18 & 0.0015 & 0.0012 \\
\hline & P. smithiana & 98 & 4,986 & 38 & 0.0015 & 0.0017 & $2,442.88$ & 17 & 0.0014 & 0.0018 & $2,534.12$ & 20 & 0.0015 & 0.0016 \\
\hline & P. likiangensis & 100 & 4,986 & 133 & 0.0053 & 0.0056 & $2,445.49$ & NA & NA & 0.0042 & $2,531.51$ & NA & NA & 0.00688 \\
\hline & P. wilsonii & 72 & 4,986 & 143 & 0.006 & 0.0054 & $2,445.25$ & 59 & 0.005 & 0.0044 & $2,531.75$ & 86 & 0.007 & 0.0064 \\
\hline \multirow[t]{4}{*}{ mtDNAs } & P. schrenkiana & 53 & 1,361 & 1 & 0.00016 & 0.00025 & 107.33 & 0 & 0 & 0 & $1,253.67$ & 1 & 0.00018 & 0.00027 \\
\hline & P. smithiana & 49 & 1,413 & 3 & 0.00048 & 0.00026 & 107.33 & 0 & 0 & 0 & $1,305.67$ & 3 & 0.00052 & 0.00028 \\
\hline & P. likiangensis & 91 & 1,367 & 5 & 0.00072 & 0.00101 & 107.33 & 0 & 0 & 0 & $1,259.67$ & 5 & 0.00078 & 0.00109 \\
\hline & P. wilsonii & 86 & 1,256 & 4 & 0.00063 & 0.00127 & 107.33 & 0 & 0 & 0 & $1,148.67$ & 4 & 0.00069 & 0.00139 \\
\hline \multirow[t]{4}{*}{ cpDNAs } & P. schrenkiana & 53 & 1,693 & 0 & 0 & 0 & - & - & - & - & - & - & - & - \\
\hline & P. smithiana & 49 & 1,567 & 4 & 0.00057 & 0.00055 & - & - & - & - & - & - & - & - \\
\hline & P. likiangensis & 91 & 1,699 & 9 & 0.00104 & 0.00039 & - & - & - & - & - & - & - & - \\
\hline & P. wilsonii & 86 & 1,704 & 1 & 0.00012 & 0.00003 & - & - & - & - & - & - & - & - \\
\hline
\end{tabular}

$n$ sample size, $L$ length in base pairs, $S$ number of segregating sites, $\theta_{W}$ Watterson's parameter, $\pi$ nucleotide diversity, $N A$ failed to compute 
Table $2 P$ values of neutrality tests at each locus as measured by MFDM tests

\begin{tabular}{lllll}
\hline Locus & P. schrenkiana & P. smithiana & P. likiangensis & P. wilsonii \\
\hline $4 C L$ & NA & 1.0000 & 0.6061 & 0.2254 \\
EBS & 1.0000 & 1.0000 & 1.0000 & 0.7887 \\
GI & 1.0000 & 1.0000 & 0.4444 & 1.0000 \\
MOO2 & 1.0000 & 0.2062 & 0.0808 & 0.3380 \\
M007D1 & 1.0000 & 1.0000 & 0.6869 & 1.0000 \\
Sb16 & 1.0000 & 0.0512 & 0.7071 & 0.3099 \\
Sb29 & 0.3389 & 1.0000 & 1.0000 & 1.0000 \\
Sb62 & 0.3729 & 0.2062 & 0.7677 & 0.5634 \\
sel364 & 1.0000 & 0.7835 & NA & 1.0000 \\
sel390 & 0.2034 & 0.5361 & 0.6869 & 0.6761 \\
$x y 1420$ & NA & NA & 1.0000 & 1.0000 \\
nad1 intron b/c c & 1.0000 & 1.0000 & 1.0000 & 1.0000 \\
$n a d 5$ intron1 & NA & NA & 0.2222 & 0.4471 \\
trnL-trnF & NA & $0.04433 *$ & 1.0000 & 1.0000 \\
trnS-trnG & NA & NA & $0.0222 *$ & NA \\
$n d h \mathrm{~K} / \mathrm{C}$ & NA & NA & 0.0223 & NA \\
\hline
\end{tabular}

$N A$ failed to compute due to insufficient variation

$* P<0.05 ; * * P<0.01$; and $* * * P<0.001$

of $P$. schrenkiana, $P$. smithiana, $P$. likiangensis, and $P$. wilsonii were obtained using approximate Bayesian computations (ABC-MCMC) methods and these simulated samples were used to calculate Bayes factors. All tests suggested the divergence between the four species to follow in the order: $P$. schrenkiana-P. smithiana versus $P$. likiangensis$P$. wilsonii, then $P$. likiangensis versus $P$. wilsonii, and $P$. schrenkiana versus $P$. smithiana. In addition, the model that included gene flow between these speciation events (Model B; Fig. 2) provided a better fit to the observed nuclear data than did the model without gene flow (model B versus model A; $\mathrm{BF}=876.2$ ). ABC analyses (Fig. 2) further estimated that the divergence between $P$. likiangensis-P. wilsonii and $P$. schrenkiana-P. smithiana occurred around 18.4 Mya (95 \% HPDI, 11.7-22.4 Mya) (Supplementary Table 6; Supplementary Fig. 3). The divergence between $P$. schrenkiana and $P$. smithiana was estimated at 5.0 Mya (95 \% HPDI, 3.98-7.27 Mya) while P. likiangensis and P. wilsonii diverged at 6.31 Mya (95 \% HPDI, 5.01-

Table $3 \Phi_{\text {ST }}$ values over all loci among four spruce species

P. schrenkiana P. smithiana P. likiangensis P. wilsonii

$\begin{array}{llll}\text { P. schrenkiana } & - & & \\ \text { P. smithiana } & 0.6312^{* * *} & - & \\ \text { P. likiangensis } & 0.5923^{* * *} & 0.54811^{* * *} & - \\ \text { P. wilsonii } & 0.5835^{* * *} & 0.5231^{* * *} & 0.2585^{* * *}\end{array}$

$* P<0.05 ; * * P<0.01 ;$ and $* * * P<0.001$
10.2 Mya). The results of ABC analyses revealed symmetric post-divergence gene flow between two ancestral clades and two pairs of the current species. We also examined gene flow between these four species using IMa2 program and our estimates (Table 4) were roughly consistent with the ABC results. In addition, large expansion population growth rate parameter ( $g$ ) values of $P$. schrenkiana (378.99), P. likiangensis (1205.29), and P. wilsonii (35.92) showed all these species may have experienced sudden and recent expansions. The small $(g)(0.0021)$ of $P$. smithiana indicated it to have had little or no growth.

\section{Discussion}

In the present study, we combined population genetic analyses from chloroplast, mitochondrial, and nuclear genomes to examine the origin and speciation model of $P$. schrenkiana and $P$. smithiana, occurring in the Central Asian Highlands and the Himalayas. Although some remote populations were not sampled due to the difficulty to access, all studied populations can represent the core distributions of two species. The sampled individuals for some populations are fewer than expected because of the failure during gene amplifying and sequencing. Therefore, we focused our discussions and implications on the divergence and speciation between two species and their closely related congeners, rather than intraspecific divergences and diversity. In both chloroplast and mitochondrial loci, we found that $P$. schrenkiana is more closely related to $P$. likiangensis than to $P$. wilsonii or P. smithiana. However, nuclear datasets suggested that $P$. schrenkiana and $P$. smithiana are more closely related to each other than to either of the other two species. Modeling and testing of the speciation series of four species not only supported this conclusion but further suggested gene flow accompanying speciation. This inconsistency of phylogenetic relationships and speciation series of the four species between three genomes may derive from two different but interacting factors: (1) different rates of the incomplete lineage sorting; (2) interspecific gene flow accompanying speciation. Our further estimates of the speciation timescales between these four species suggest that their initial and further divergences seem to be correlated with the early QTP uplifts and the corresponding TSMs depressions.

Incomplete Lineage Sorting and Interspecific Gene Flow Three previous studies, each mainly based on chloroplast, mitochondrial, or nuclear sequence variations, but from fewer individuals, discovered a close affinity of $P$. schrenkiana with P. likiangensis (Ran et al. 2006) or $P$. wilsonii (Lockwood et al. 2013) or P. smithiana (Sun et al. 2014). In the present study, our phylogenetic analyses of both chloroplast and mitochondrial sequence variations suggest that $P$. schrenkiana seems to be more closely related to $P$. likiangensis (Fig. 4 ) than to $P$. smithiana. The 
Fig. 5 Structure analysis of four species when $K=2-4$ clusters are assumed. For each $K$ value, results of the run with the highest value of LnPD were used.

Variation among runs was limited

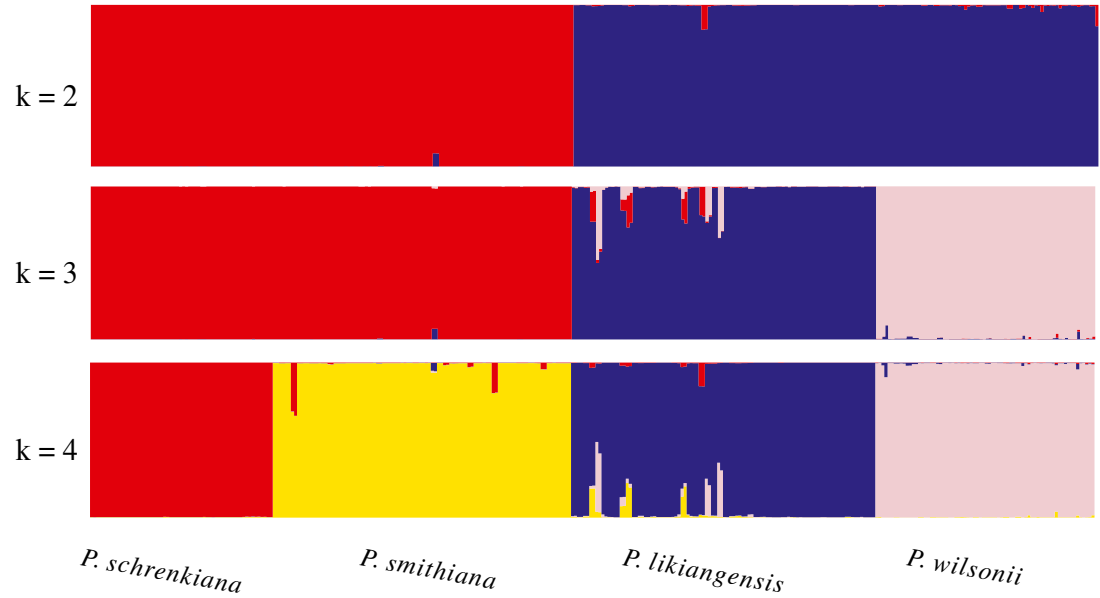

clade containing haplotypes from these three species comprise a distinct clade that differs from that comprising the $P$. wilsonii haplotypes. However, both phylogenetic and population genetic analyses based on nuclear genetic sequence data suggest that $P$. schrenkiana and $P$. smithiana cluster first, while $P$. likiangensis and $P$. wilsonii together form the other clade (Fig. 6). It should be noted that within the latter clade, individuals from two species did not cluster into separate subclades. This would have resulted from substructures of each species and interspecific gene flow or introgressions, which may have distorted phylogenetic analyses of population data. In fact, STRUCTURE analysis clearly supported these two distinct species although gene flow and introgression between them were detected for a few individuals (Fig. 5). The divergence series between four species were further confirmed by modeling and testing of the genetic population data from the nuclear loci (Figs. 2 and 6). The inconsistent interspecific relationships at different genomes may have been caused by the following two factors. First, In contrast to the biparental

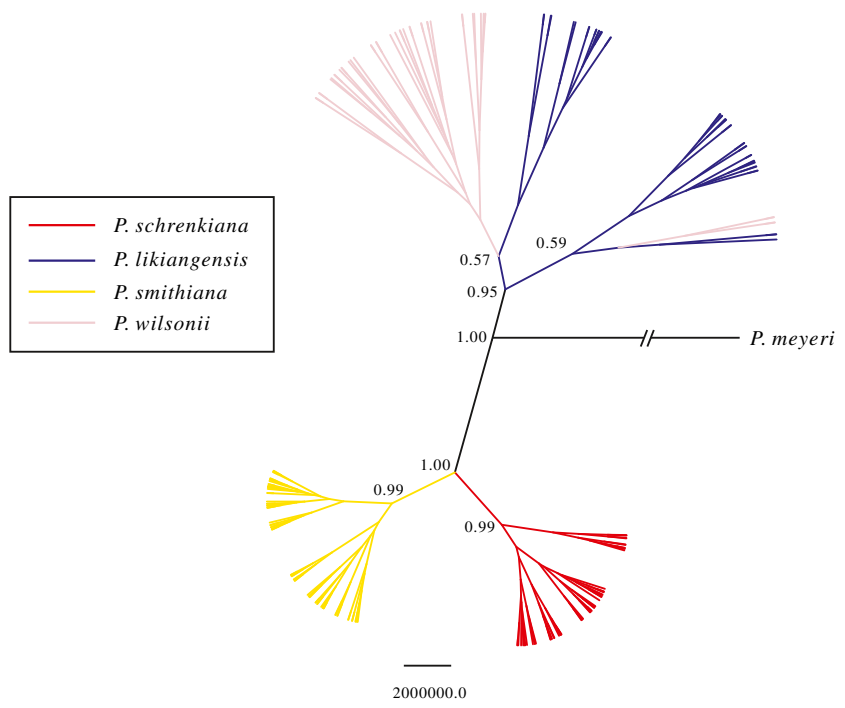

Fig. 6 The phylogenetic tree based on the concatenated nuclear data using the BEAST 1.7.2. The color of each line indicates each species, and the length of each line shows the genetic distance inheritance exhibited by nuclear DNA (nrDNA), mtDNA is maternally transmitted through seeds, while cpDNA is paternally inherited via pollen in spruce species (Corriveau and Coleman 1988; Harris and Ingram 1991; Reboud and Zeyl 1994); therefore, the average coalescence time of two randomly picked chloroplast or mitochondrial alleles $\left(N_{\mathrm{e}}\right.$ generation) is half of the nuclear DNA alleles $\left(2 N_{\mathrm{e}}\right.$ generation; Nei and Tajima 1981). Therefore, DNA variation would be lost more easily and species-specific mutations would be accumulated more quickly in cytoplasmic DNAs than in nuclear DNAs, especially in extremely small isolated populations. This may account for speciesspecific haplotypes in these two cytoplasmic DNAs. For $P$. smithiana which was isolated with other species by Himalayas, the small effective population size may have led to a high level of accumulation of species-specific mutations in the cytoplasmic DNA, which probably distorted the true interspecific relationships in the results of earlier studies (Ran et al. 2006;

Table 4 Maximum-likelihood estimates (MLE) and the $95 \%$ highest posterior density (HPD) Intervals of migration rate among pair-wise comparisons

\begin{tabular}{lllll}
\hline & & MLE & HPD95Lo & HPD95Hi \\
\hline P. schrenkiana/P. smithiana & $m_{1}$ & 0.079 & 0 & 1.763 \\
& $m_{2}$ & 0.001 & 0 & 1.615 \\
P. schrenkiana/P. likiangensis & $m_{1}$ & 0.175 & 0 & 0.593 \\
& $m_{2}$ & 0.001 & 0 & 0.427 \\
P. schrenkiana/P. wilsonii & $m_{1}$ & 0.0645 & 0 & 0.3615 \\
& $m_{2}$ & 0.0015 & 0 & 0.6345 \\
P. smithiana/P. likiangensis & $m_{1}$ & 0.1375 & 0 & 0.8425 \\
& $m_{2}$ & 0.0025 & 0 & 1.127 \\
P. smithiana/P. wilsonii & $m_{1}$ & 0.1005 & 0 & 0.4995 \\
& $m_{2}$ & 0.0045 & 0 & 0.6885 \\
P. likiangensis/P. wilsonii & $m_{1}$ & 0.005 & 0 & 0.805 \\
& $m_{2}$ & 0.445 & 0.055 & 1.325 \\
\hline
\end{tabular}

$m_{1}$ is the migration rate from species 1 to species 2 forward in time, and $m_{2}$ is the migration from species 2 to species 1 
Bouillé et al. 2011; Zou et al. 2013). However, at the nuclear loci, incomplete lineage sorting still remains for most sampled loci. The additive mutations from multiple nuclear loci therefore recovered a different interspecific relationship from those based on mitochondrial and chloroplast sequence variations. Second, gene flow and interspecific introgressions, especially cytoplasmic haplotypes occur frequently in spruce ( $\mathrm{Li}$ et al. 2010; Du et al. 2011; Zou et al. 2012). It is therefore likely that $P$. schrenkiana captured mitochondrial and chloroplast haplotypes from P. likiangensis. In addition, modeling of the nuclear population genetic data suggests that gene flow continued for a long time after the divergence. Gene flow at nuclear loci maybe occured more frequently between $P$. schrenkiana and $P$. smithiana than between $P$. wilsonii and/or P. likiangensis (Supplementary Table 6). Therefore, it is also likely the regional nuclear gene flow might have grouped $P$. schrenkiana and $P$. smithiana together. All these lines of evidence suggested that speciation histories of these species are more complex than expected and that both incomplete lineage sorting and gene flow might have together resulted in different interspecific relationships discovered between cytoplasmic and nuclear genomes.

Geological Isolation and Speciation History According to our parameter estimates based on $4 \times 10^{6}$ simulated samples and the current data pattern, two clades $P$. schrenkiana-P. smithiana and P. likiangensis $-P$. wilsonii diverged around 18.4 Mya. The further divergences between $P$. likiangensis and $P$. wilsonii and between P. schrenkiana and P. smithiana were estimated to occur at 6.31 and 5.0 Mya, respectively. It is interesting to note that the divergence timescales estimated here agree well with the geological evidence that the QTP (and Himalayas) were uplifted extensively during these stages. For example, the first large-scale QTP-Himalayas uplifts were dated to occur at $22 \mathrm{Mya}$, although the uplift of the QTP and Himalayas began about 50 Mya, which caused some peaks that were high enough to trigger Asian desertification (Guo et al. 2002). Between 10 and 8 Mya, the second uplift might have occurred, which further enhanced the aridity of the Asian interior and the onset of the Asian monsoon (An et al. 2001). This uplift may have occurred as early as $15 \mathrm{Mya}$, and in particular may have caused further increases of the western Kunlun Mountains, the Himalayas, and the vast depressions of the Tarim (Wang et al. 1990; Abdrakhmatov et al. 1996; Shi et al. 1998; An et al. 2001; Bullen et al. 2001, 2003; Charreau et al. 2006, 2009; Wang et al. 2006; Buslov et al. 2007; Dupont-Nivet et al. 2008; Wang et al. 2008). It is also likely that the first extensive uplift promoted the initial divergence between two clades, $P$. likiangensis $-P$. wilsonii and $P$. schrenkiana-P. smithiana. It is interesting to find this divergence was accompanied with gene flow according to the selected fitter model. The second large-scale uplift might have terminated this gene flow and also initiated further divergences between $P$. likiangensis and $P$. wilsonii and between $P$. schrenkiana and $P$. smithiana. The TSMs deserts formed at this stage (Sun et al. 2009) and the further increase of the Himalayas should have restricted natural migration or dispersal between P. schrenkiana and P. smithiana (Fig. 1). Therefore, these two extensive uplifts of the QTP (-Himalayas) might have played an important role in the speciation histories of these four species.

In addition to previous studies, which have demonstrated that several herbal genera originated or diversified greatly during the QTP uplift stages (e.g., Liu et al. 2002, 2006; Wang et al. 2009), our population genetic analyses have indicated that trees with long generation times may also have speciated in response to these uplifts. Further analyses of more tree speciation events in the QTP and adjacent regions are needed to demonstrate this generality. Overall, these findings together highlight the complex speciation histories of the alpine plants, and the importance of the geological events in promoting plant diversification.

Acknowledgments The authors thank Dr. Kun Wang, Long Li, and Ting $\mathrm{Xu}$ for their helps in analyzing data. This research was supported by grants from National Key Project for Basic Research (2014CB954100), National Natural Science Foundation of China (30930072), Key Project of International Collaboration Program, Ministry of Science and Technology of China (2010DFB63500) and '111' collaboration Program.

Open Access This article is distributed under the terms of the Creative Commons Attribution License which permits any use, distribution, and reproduction in any medium, provided the original author(s) and the source are credited.

\section{References}

Abdrakhmatov KY, Aldazhanov SA, Hager BH, Hamburger MW, Herring TA, Kalabaev KB, Makarov VI, Molnar P, Panasyuk SV, Prilepin MT, Reilinger RE, Sadybakasov IS, Souter BJ, Trapeznikov YA, Tsurkov VY, Zubovich AV (1996) Relatively recent construction of the Tien Shan inferred from GPS measurements of presentday crustal deformation rates. Nature 384:450-453

An ZS, Kutzbach JE, Prell WL, Port SC (2001) Evolution of Asian monsoons and phased uplift of the Himalayan-Tibetan plateau since late Miocene times. Nature 411:62-66

Anderson LL, Hu FS, Nelson DM, Petit RJ, Paige KN (2006) Ice-age endurance: DNA evidence of a white spruce refugium in Alaska. Proc Natl Acad Sci U S A 103:12447-12450

Bandelt HJ, Forster P, Röhl A (1999) Median-joining networks for inferring intraspecific phylogenies. Mol Biol Evol 16:37-48

Bouillé M, Senneville S, Bousquet JB (2011) Discordant mtDNA and cpDNA phylogenies indicate geographic speciation and reticulation as driving factors for the diversification of the genus Picea. Tree Genet Genomes 7:469-484

Bousquet J, Bouillé M (2005) Trans-species shared polymorphism at orthologous nuclear gene loci among distant species in the conifer Picea (Pinaceae): implications for the long-term maintenance of genetic diversity in trees. Am J Bot 92:63-73

Bullen ME, Burbank DW, Garver JI, Abdrakhmatov KY (2001) Late Cenozoic tectonic evolution of the northwestern Tien Shan: new age estimates for the initiation of mountain building. Geol Soc Am Bull 113:1544-1559 
Bullen ME, Burbank DW, Garver JI (2003) Building the northern Tien Shan: integrated thermal, structural, and topographic constraints. J Geol 111:149-165

Burns RM, Honkala BH (1990) Silvics of North America. Vol 1 conifers. agriculture handbook. U.S. Department of Agriculture and Forestry Service, Washington, p 654

Buslov MM, Grave JD, Bataleva EAV, Batalev VY (2007) Cenozoic tectonic and geodynamic evolution of the Kyrgyz Tien Shan mountains: a review of geological, thermochronological and geophysical data. J Asian Earth Sci 29:205-214

Charreau J, Gilder S, Chen Y, Dominguez S, Avouac JP, Sen S, Jolivet M, Li YG, Wang WM (2006) Magnetostratigraphy of the Yaha Section, Tarim basin (China): 11 Ma acceleration in erosion and uplift of the Tian Shan mountains. Geology 34:181-184

Charreau J, Chen Y, Gilder S, Barrier L, Domingguez S, Augier R, Sen S, Avouac JP, Gallaud A, Graveleau F, Wang QC (2009) Neogene uplift of the Tian Shan Mountains observed in the magnetic record of the Jingou River section (northwest China). Tectonics 28:1-22

Chen J, Källman T, Gyllenstrand N, Lascoux M (2010) New insights on the speciation history and nucleotide diversity of three boreal spruce species and a Tertiary relict. Heredity 104:3-14

Corriveau JL, Coleman AW (1988) Rapid screening method todetect potential biparental inheritance of plastid DNA and results for over 200 angiosperm species. Am J Bot 75:1443-1458

Coyne JA (1992) Genetics and speciation. Nature 355:511-515

Coyne JA, Orr HA (2004) Speciation. Sinauer, Sunderland, MA

Doyle JJ, Doyle JL (1990) Isolation of plant DNA from plant tissue. Focus 12:13-15

Drummond AJ, Rambaut A (2007) BEAST: Bayesian evolutionary analysis by sampling trees. BMC Evol Biol 7:214

Du FK, Peng XL, Liu JQ, Lascou M, Hu FS, Petit RJ (2011) direction and extent of organelle DNA introgression between two spruce species in the Qinghai-Tibetan Plateau. New Phytol 192:1024-1033

Dupont-Nivet G, Hoorn C, Konert M (2008) Tibetan uplift prior to the eocene-oligocene climate transition: evidence from pollen analysis of the Xining basine transition. Geology 6:987-990

Evanno G, Regnaut S, Goudet J (2005) Detecting the number of clusters of individuals using the software structure: a simulation study. Mol Ecol 14:2611-2620

Ewing B, Hillier L, Wendl MC, Green P (1998) Base-calling of automated sequencer traces using phred. I. accuracy assessment. Genome Res 8:175-185

Excoffier L, Foll M (2011) fastsimcoal: a continuous-time coalescent simulator of genomic diversity under arbitrarily complex evolutionary scenarios. Bioinformatics 27:1332-1334

Excoffier L, Laval G, Schneider S (2005) Arlequin ver. 3.0: an integrated software package for population genetics data analysis. Evol Bioinforma 1:47-50

Farjón A (2001) World checklist and bibliography of conifers. Royal Botanic Gardens, Kew

Fu L, Li N, Mill RR (1999) Picea. In: Wu, ZY, Raven, PH (eds.). Flora of China. Science Press, Beijing, pp 25-32

Gavrilets S (2003) Perspective: models of speciation: what have we learned in 40 years? Evolution 57:2197-2215

Grant V (1981) Plant speciation. Columbia University Press, New York

Guo ZT, Ruddiman WF, Hao QZ, Wu HB, Qiao YS, Zhu RX, Peng SZ, Wei JJ, Yuan BY, Liu TS (2002) Onset of Asian desertification by 22 Mya ago inferred from loess deposits in China. Nature 416:159163

Hailer F, Kutschera VE, Hallström BM, Klassert SR, Leonard JA, Arnason U, Janke A (2012) Nuclear genomic sequences reveal that polar bears are an old and distinct bear lineage. Science 336:44-347
Hamilton MB (1999) Four primer pairs for the amplification of chloroplast intergenic regions with intraspecific variation. Mol Ecol 8: 521-523

Harris SA, Ingram R (1991) Chloroplast DNA and biosystematics, the effects of intraspecific diversity and plastid transmission. Taxon 40: 393-412

Hein J, Schierup MH, Wiuf C (2004) Gene genealogies, variation and evolution, a primer in coalescent theory. Oxford University Press, Oxford

Heuertz M, Paoli ED, Kallman T, Larsson H, Jurman M, Morgante M, Lascoux M, Gyllenstrand N (2006) Multilocus patterns of nucleotide diversity, linkage disequilibrium and demographic history of Norway spruce (Picea abies (L.) Karst). Genetics 174:2095-2105

Hey J (2006) Recent advances in assessing gene flow between diverging populations and species. Curr Opin Genet Dev 16:592-596

Hey J (2010a) Isolation with migration models for more than two populations. Mol Biol Evol 27:905-920

Hey J (2010b) The divergence of chimpanzee species and subspecies as revealed in multipopulation isolation-with-migration analyses. Mol Biol Evol 27:921-933

Hey J, Nielsen R (2004) Multilocus methods for estimating population sizes, migration rates and divergence time, with applications to the divergence of Drosophila pseudoobscura and D. persimilis. Genetics 167:747-760

Hey J, Nielsen R (2007) Integration within the Felsenstein equation for improved Markov chain Monte Carlo methods in population genetics. Proc Natl Acad Sci U S A 104:2785-2790

Hubisz MJ, Falush D, Stephens M, Pritchard JK (2009) Inferring weak population structure with the assistance of sample group information. Mol Ecol Resour 9:1322-1332

Huelsenbeck JP, Ronquist F, Nielsen R, Bollback JP (2001) Bayesian inference of phylogeny and its impact on evolutionary biology. Science 294:2310-2314

Kuhner MK (2006) LAMARC 2.0: maximum likelihood and Bayesian estimation of population parameters. Bioinformatics 22:768-770

Levin D (2003) The ecological transition in speciation. New Phytol 161: 91-96

Li HP (2011) A new test for detecting recent positive selection that is free from the confounding impacts of demography. Mol Biol Evol 28: 365-375

Li N, Stephens M (2003) Modeling linkage disequilibrium and identifying recombination hotspots using single-nucleotide polymorphism data. Genetics 165:2213-2233

Li Y, Stocks M, Hemmilä S, Källman T, Zhu HT, Zhou YF, Chen J, Liu JQ, Lascoux M (2010) Demographic histories of four spruce (Picea) species of the Qinghai-Tibetan Plateau and neighboring areas inferred from multiple nuclear loci. Mol Biol Evol 27:1001-1014

Li L, Abbott RJ, Liu BB, Sun YS, Li LL, Zou JB, Wang X, Miehe G, Liu JQ (2013) Pliocene intraspecific divergence and Plio-Pleistocene range expansions within Picea likiangensis (Lijiang spruce), a dominant forest tree of the Qinghai-Tibet Plateau. Mol Ecol 22:52375255

Librado P, Rozas J (2009) DnaSP v5: a software for comprehensive analysis of DNA polymorphism data. Bioinformatics 25:1451-1452

Liu TS (1982) A new proposal for the classification of the genus Picea. Acta Phytol Geobot 33:227-244

Liu JQ, Gao TG, Chen ZD, Lu AM (2002) Molecular phylogeny and biogeography of the Qinghai-Tibet Plateau endemic Nannoglottis (Asteraceae). Mol Phylogenet Evol 23:307-325

Liu JQ, Wang YJ, Wang AL, Hideaki O, Abbott RJ (2006) Radiation and diversification within the Ligularia-Cremanthodium-Parasenecio complex (Asteraceae) triggered by uplift of the Qinghai-Tibetan Plateau. Mol Phylogenet Evol 38:31-49

Lockwood JD, Jelena MA, Zou JB, Wang J, Liu JQ, Susanne SR (2013) A new phylogeny for the genus Picea from plastid, mitochondrial, and nuclear sequences. Mol Phylogenet Evol 60:717-727 
Mao KS, Hao G, Liu JQ, Adams RP, Milne RI (2010) Diversification and biogeography of Juniperus (Cupressaceae): variable diversification rates and multiple intercontinental dispersals. New Phytol 188:254 272

Mayr E (1954) Change of genetic environment and evolution. Columbia University Press, New York

Meng L, Yang R, Abbott RJ, Miehe G, Hu TH, Liu JQ (2007) Mitochondrial and chloroplast phylogeography of Picea crassifolia Kom. (Pinaceae) in the Qinghai-Tibetan Plateau and adjacent highlands. Mol Ecol 16:4128-4137

Myers N, Mittermeier RA, Mittermeier CG, da Fonseca GAB, Kent J (2000) Biodiversity hotspots for conservation priorities. Nature 403: $853-858$

Nei M, Tajima F (1981) Genetic drift and estimation of effective population size. Genetics 98:625-640

Nielsen R, Wakeley J (2001) Distinguishing migration from isolation: a Markov chain Monte Carlo approach. Genetics 158:885-896

Orr HA, Presgraves DC (2000) Speciation by postzygotic isolation, forces, genes and molecules. Bioessays 22:1085-1094

Posada D (2008) JModelTest: phylogenetic model averaging. Mol Biol Evol 25:1253

Pritchard JK, Stephens M, Donnelly P (2000) Inference of population structure using multilocus genotype data. Genetics 155:945-959

Ran JH, Wei XX, Wang XQ (2006) Molecular phylogeny and biogeography of Picea (Pinaceae): implication for phylogeographical studies using cytoplasmic haplotypes. Mol Phylogenet Evol 41:405-419

Reboud X, Zeyl C (1994) Organelle inheritance in plants. Heredity 72 : $132-140$

Rieseberg LH, Willis JH (2007) Plant speciation. Science 317:910-914

Ronquist F, Huelsenbeck JP (2003) MrBayes 3: Bayesian phylogenetic inference under mixed models. Bioinformatics 19:1572-1574

Rosenberg NA (2004) Distruct: a program for the graphical display of population structure. Mol Ecol Notes 4:137-138

Shi YF, Li JJ, Li BY (1998) Uplift and environmental changes of Qinghai-Xizang Plateau in the Late Cenozoic. Guangdong Science \& Technology Press, Guangzhou, p 154

Stephens M, Donnelly P (2003) A comparison of Bayesian methods for haplotype reconstruction from population genotype data. Am J Hum Genet 73:1162-1169

Stephens M, Smith NJ, Donnelly P (2001) A new statistical method for haplotype reconstruction from population data. Am J Hum Genet 68:978-989

Sun J, Zhang Z, Zhang L (2009) New evidence on the age of the Taklimakan desert. Geology 37:159-162

Sun YS, Abbott JR, Li LL, Zou JB, Liu JQ (2014) Evolutionary history of purple cone spruce (Picea Purpurea) in the Qinghai-Tibet Plateau: homoploid hybrid origin and Pleistocene expansion. Mol Ecol 23(2):343-359. doi:10.1111/Mec.12599

Taberlet P, Gielly L, Pautou G, Bouvet J (1991) Universal primers for amplification of three non-coding regions of chloroplast DNA. Plant Mol Biol 17:1105-1109

Tajima F (1983) Evolutionary relationship of DNA sequences in finite populations. Genetics 105:437-460

Tajima F (1989) Statistical method for testing the neutral mutation hypothesis by DNA polymorphism. Genetics 123:585-595
Takayama K, Sun BY, Stuessy TF (2013) Anagenetic speciation in Ullung Island, Korea: genetic diversity and structure in the island endemic species, Acer takesimense (Sapindaceae). J Plant Res 126: 323-333

Tamura K, Peterson D, Peterson N, Stecher G, Nei M, Kumar S (2011) MEGA5: molecular evolutionary genetics analysis using maximum likelihood, evolutionary distance, and maximum parsimony methods. Mol Biol Evol 28:2731-2739

Thompson JD, Gibson TJ, Plewniak F, Jeanmougin F, Higgins DG (1997) The CLUSTAL X windows interface: flexible strategies for multiple sequence alignment aided by quality analysis tools. Nucleic Acids Res 25:4876-4882

Wakeley J (2003) Inferences about the structure and history of populations: coalescents and intraspecific phylogeography. In: Singh R, Veuille M (eds) The evolution of population biology. Cambridge University Press, New York

Wang D, Sun X, Zhao Y (1990) Late cretaceous to tertiary palynofloras in Xinjiang and Qinghai, china. Rev Palaeobot Palynol 65:95-104

Wang Y, Deng T, Biasatti D (2006) Ancient diets indicate significant uplift of southern Tibet after ca. 7 Ma. Geology 34:309-312

Wang CS, Zhao XX, Liu ZF, Lippert PC, Graham SA, Coe RS, Yi HS, Zhu LD, Liu S, Li YL (2008) Constraints on the early uplift history of the Tibetan Plateau. Proc Natl Acad Sci U S A 105:4987-4992

Wang LY, Abbott RJ, Zheng W, Chen P, Wang YJ, Liu JQ (2009) History and evolution of alpine plants endemic to the Qinghai-Tibet Plateau: Aconitum gymnandrum (Ranunculaceae). Mol Ecol 18:709-721

Watterson GA (1975) On the number of segregating sites in genetical models without recombination. Theor Popul Biol 7:256-276

Wegmann D, Leuenberger C, Excoffier L (2009) Efficient approximate Bayesian computation coupled with Markov chain Monte Carlo without likelihood. Genetics 182:1207-1218

Wegmann D, Leuenberger C, Neuenschwander S, Excoffier L (2010) ABCtoolbox: a versatile toolkit for approximate Bayesian computations. BMC Bioinforma 11:116-122

Wilson EO (1992) The diversity of life. Harvard University Press, Cambridge

Woerner AE, Cox MP, Hammer MF (2007) Recombination-filtered genomic datasets by information maximization. Bioinformatics 23 : $1851-1853$

Xu TT, Abbott R, Milne R, Mao KS, Du FK, Wu GL, Ciren ZX, Miehe G, Liu JQ (2010) Phylogeography and allopatric divergence of cypress species (Cupressus L.) in the Qinghai-Tibetan Plateau and adjacent regions. BMC Evol Biol 10:194-203

Zhang YS, Tang GC (1989) Picea schrenkiana forest. In: Editorial Committee of Xinjiang Forest (ed) Xinjiang Forest. Xinjiang People Press, Urumqi, pp 121-149

Zou JB, Peng XL, Li L, Liu JQ, Miehe G, Opgenoorth L (2012) Molecular phylogeography and evolutionary history of Picea likiangensis in the Qinghai-Tibetan Plateau inferred from mitochondrial and chloroplast DNA sequence variation. J Syst Evol 50:341350

Zou JB, Sun YS, Li L, Wang GN, Yue W, Lu ZQ, Wang Q, Liu JQ (2013) Population genetic evidence for speciation patter and gene flow between Picea wilsonii, P. morrisonicola and P. neoveitchii. Ann Bot 12:1829-1844 